\title{
Correction to: Association between dietary acid load and the risk of hypertension among adults from South China: result from nutrition and health survey (2015-2017)
}

Shao-wei Chen, Gui-yuan Ji, Qi Jiang, Ping Wang, Rui Huang, Wen-jun Ma, Zi-hui Chen ${ }^{*}$ and Jie-wen Peng*

\section{Correction to: BMC Public Health (2019) 19:1599 https://doi.org/10.1186/s12889-019-7985-5}

It was highlighted that in the original article [1] the data on categorical variables was wrongly arranged in Table 1 and there was an incorrect statement in the fourth paragraph of the Discussion section. This Correction article shows the incorrect and correct statement of paragraph 4 and the correct Table 1.

\section{Incorrect statement}

Even though the prevalence rate of hypertension in male $(29.1 \%)$ was mildly lower than female $(32.7 \%)$, our finding showed a significantly higher PRAL in the male $(25.3 \pm 20.2 \mathrm{mEq} / \mathrm{d})$ comparing to the female $(19.4 \pm$ $16.7 \mathrm{mEq} / \mathrm{d})$.

\section{Correct statement}

Prevalence rate of hypertension in male $(32.7 \%)$ was mildly higher than female $(29.1 \%)$ and a significantly higher PRAL in the male $(25.3 \pm 20.2 \mathrm{mEq} / \mathrm{d})$ was also identified comparing to the female $(19.4 \pm 16.7 \mathrm{mEq} / \mathrm{d})$.
Published online: 19 March 2020

Reference

1. Chen SW, et al. Association between dietary acid load and the risk of hypertension among adults from South China: result from nutrition and health survey (2015-2017). BMC Public Health. 2019;19:1599. https://doi.org/ $10.1186 /$ s12889-019-7985-5.

*Correspondence: gdpiph2017@163.com; gdpiph2017@163.com Department of Health Risk Assessment Research Center, Guangdong Provincial Institute of Public Health, Guangdong Provincial Center for Disease Control and Prevention, No. 160 Qunxian Road, Panyu District, Guangzhou 511430, China

(c) The Author(s). 2020 Open Access This article is licensed under a Creative Commons Attribution 4.0 International License, which permits use, sharing, adaptation, distribution and reproduction in any medium or format, as long as you give appropriate credit to the original author(s) and the source, provide a link to the Creative Commons licence, and indicate if changes were made. The images or other third party material in this article are included in the article's Creative Commons licence, unless indicated otherwise in a credit line to the material. If material is not included in the article's Creative Commons licence and your intended use is not permitted by statutory regulation or exceeds the permitted use, you will need to obtain permission directly from the copyright holder. To view a copy of this licence, visit http://creativecommons.org/licenses/by/4.0/. The Creative Commons Public Domain Dedication waiver (http://creativecommons.org/publicdomain/zero/1.0/) applies to the data made available in this article, unless otherwise stated in a credit line to the data. 
Table 1 Characteristic of eligible participants

\begin{tabular}{|c|c|c|c|}
\hline & Overall $(n=3501)$ & Male $(n=1608)$ & Female $(n=1893)$ \\
\hline Age (years) & $52.0 \pm 15.0$ & $52.9 \pm 15.2$ & $51.2 \pm 14.7$ \\
\hline \multicolumn{4}{|l|}{ Ethnicity (N, \%) } \\
\hline Han & 3473 (99.2) & 1599 (99.4) & $1874(99.0)$ \\
\hline Other & $28(0.8)$ & $9(0.6)$ & $19(1.0)$ \\
\hline BMI (Kg/m2) & $23.4 \pm 3.5$ & $23.3 \pm 3.4$ & $23.4 \pm 3.6$ \\
\hline \multicolumn{4}{|l|}{ Nutrient intake } \\
\hline Protein (g/d) & $65.9 \pm 36.5$ & $71.5 \pm 40.4$ & $61.1 \pm 32.0$ \\
\hline Calcium (mg/d) & $1501.6 \pm 603.9$ & $1564.6 \pm 579.3$ & $1448.1 \pm 619.1$ \\
\hline Potassium (mg/d) & $381.1 \pm 211.1$ & $389.4 \pm 184.7$ & $374.1 \pm 231.0$ \\
\hline Phosphorus (mg/d) & $866.6 \pm 298.7$ & $926.2 \pm 298.9$ & $815.9 \pm 289.1$ \\
\hline Magnesium (mg/d) & $238.4 \pm 82.3$ & $250.7 \pm 83.3$ & $228.0 \pm 80.0$ \\
\hline Sodium (mg/d) & $4893.5 \pm 3366.0$ & $5228.2 \pm 3400.4$ & $4609.2 \pm 3310.9$ \\
\hline Total energy intake (Kcal/d) & $1757.4 \pm 566.5$ & $1908.6 \pm 604.8$ & $1629.0 \pm 497.1$ \\
\hline PRAL (mEq/d) & $22.1 \pm 18.6$ & $25.3 \pm 20.2$ & $19.4 \pm 16.7$ \\
\hline $\operatorname{NEAP}(\mathrm{mEq} / \mathrm{d})$ & $86.8 \pm 53.9$ & $90.4 \pm 58.9$ & $83.8 \pm 49.1$ \\
\hline $\mathrm{DBP}(\mathrm{mmHg})$ & $131.0 \pm 21.2$ & $132.6 \pm 20.1$ & $129.6 \pm 22.0$ \\
\hline SBP $(\mathrm{mmHg})$ & $77.4 \pm 11.5$ & $79.1 \pm 11.3$ & $75.9 \pm 11.5$ \\
\hline Sedentary leisure time $(\mathrm{h} / \mathrm{d})$ & $4.9 \pm 2.8$ & $5.1 \pm 2.8$ & $4.8 \pm 2.7$ \\
\hline Physical activity time (h/d) & $1.1 \pm 0.8$ & $1.1 \pm 0.8$ & $1.0 \pm 0.7$ \\
\hline Hypertension (N, \%) & $1076(30.7)$ & $526(32.7)$ & $550(29.1)$ \\
\hline Smoking (N, \%) & $955(27.3)$ & $904(56.2)$ & $51(2.7)$ \\
\hline Alcohol (N, \%) & $1330(38.0)$ & $857(53.3)$ & $473(25.0)$ \\
\hline \multicolumn{4}{|l|}{ Education (N, \%) } \\
\hline$\leq 6$ years & $1674(47.8)$ & $612(38.1)$ & $1062(56.1)$ \\
\hline $7-12$ years & $1482(43.3)$ & $924(57.5)$ & $761(40.2)$ \\
\hline$\geq 13$ years & $345(9.9)$ & $72(4.5)$ & $70(3.7)$ \\
\hline \multicolumn{4}{|l|}{ Marital status (N, \%) } \\
\hline Unmarried & $329(9.4)$ & $151(9.4)$ & $178(9.4)$ \\
\hline Married & 3172 (90.6) & 1457 (90.6) & 1715 (90.6) \\
\hline
\end{tabular}

Footprint: $B M I$ body mass index, PRAL potential renal acid load, NEAP net endogenous acid production, SBP systolic blood pressure, DBP diastolic blood pressure 